\title{
PHYSIOLOGICAL DIFFERENCES BETWEEN PREPUBERTAL AND PUBERTAL SWIMMERS IN PERFORMING AN ANAEROBIC EFFORT
}

\author{
Adrian RĂDULESCU ${ }^{1,2}$, Gheorghe MARINESCU' ${ }^{1}$, Laurențiu Daniel TICALĂ ${ }^{1}$ \\ ${ }^{1}$ National University of Physical Education and Sport, Faculty of Physical Education and Sport, Bucharest, Romania \\ ${ }^{2}$ Aqua Team Sports Club, Bucharest, Romania \\ *Corresponding author: adrian.radulescu90@yahoo.com
}

DOI: $10.35189 /$ iphm.icpesk.2019.36

\begin{abstract}
Anaerobic glycolysis represents an effective means of energy production only during short and intense exercise, considering that it provides energy for a period ranging from 10 seconds to 2 minutes; hence, the aerobic effort capacity has a significant impact on the results in swimming competitions. We conducted this research to compare the physiological responses of prepubertal and pubertal swimmers to a high-intensity effort that would highlight anaerobic metabolism in blood lactate measurement. Blood lactate measurements, as well as mathematical and statistical data analysis, were used in order to create a better profile of anaerobic effort for young swimmers. The research subjects were required to swim a 100-meter freestyle time trial. Blood lactate values were recorded before and after the high-intensity swim effort in the first, fifth minute and twelfth minute. We compared blood lactate measurements and observed that, in the first minute, the difference was $0.3 \%$ between the two groups. In the fifth minute, the difference grew, and prepubertal swimmers had a larger value with a substantial margin of $11.53 \%$. The third measurement showed that the prepubertal group had a smaller value with $3.91 \%$. From this perspective, the results of both groups indicate to some extent a similar physiological response when swimming a high-intensity anaerobic time trial.
\end{abstract}

Keywords: anaerobic capacity, blood lactate, young swimmers.

\section{Introduction}

Many different specialists in sport science consider the aerobic effort capacity to be the only relevant energy pathway that young swimmers should train if they aim to achieve long-term success.

For all that, the anaerobic capacity makes a tremendous difference in the outcome of every race.

Our research aimed to find similarities and differences between the physiological responses of prepubertal and pubertal swimmers to an anaerobic effort.

The swimmers performed a 100-meter freestyle time trial and their blood lactate values were gathered with an analyser. The data were afterwards processed using mathematical and statistical methods.

We sampled and recorded the blood lactate values before the test and after, it in the first, fifth and twelfth minute. Calculations showed that, in the first minute, the difference was $0.3 \%$ between the two groups, indicating a nearly identical reaction of the physiological value. There was a difference of $11.53 \%$ in the fifth minute between the two groups, with the prepubertal swimmers having the larger value. The third measurement showed that the prepubertal group had a smaller value with $3.91 \%$.

We think that the results show a likewise physiological response from both groups of swimmers. The blood lactate values highlight a slightly different dynamics at the five minute mark, which subsides in the twelfth minute.

\section{Problem statement}

Age-group training represents a key sequence in the long-term development of successful junior and senior swimmers. Long-term development should be a thoroughly guided process that provides meaningful learning experiences for the swimmer.

After the first years of training, which mostly focus on skill acquisition and the learning of all four strokes, the swimmers should enter a second phase of training that Sweetenham and Atkinson (2003) call "learning-to-train phase". This phase overlaps with most of the prepubertal and pubertal period that a swimmer will experience. It is a phase that turns the focus to "extending the volume of training progressively" because "training volumes become a critical factor in long-term development". Skills and technique continue to be important aspects of training, but they are related to "the progressively greater seasonal loads". However, this does not mean that "speed is ignored, but weight is given to the volume of training with respect to the portion of the training that is intense". (Sweetenham \& Atkinson, 2003, p. 209)

When it comes to speed and competitive swimming, we must refer to anaerobic metabolism as the first pathway in which the young swimmers' bodies produce the required energy. 
Stager, Stickford and Grand (2015) define anaerobic metabolism as "the predominant energy pathway if the amount of muscular work is such that the energy requirements are greater than what can be supplied aerobically" (p. 158), adding that there are two anaerobic pathways, anaerobic glycolysis playing the most significant role because it "is an effective means of energy production during short, intense exercise, providing energy for a period ranging from 10 seconds to 2 minutes" (p. 159).

Brooks (2011) claims that "sprinting capacity is generally linked with maturation, skinny young swimmers at early stages of their physical development are not yet built for sprinting" and mentions that "young swimmers respond aerobically to all kinds of physiological stressors, so precise energy-systems work is relatively pointless" (p. 144). He concludes that "specificity does not apply to the extent that it does with mature athletes" and that high-intensity anaerobic training will not evoke the same training response in age-groupers as it will in senior athletes" (Brooks, 2011, p. 129).

Maglischo (1993) addresses the issue of the anaerobic capacity of age-group swimmers and states that "the anaerobic capacity of children is lower than that of adults, even when it is measured relative to their size" (p. 253). $\mathrm{He}$ further explains that "anaerobic capacity increases at the time of puberty, with maximum blood lactate increasing between the ages of 12 and 13 until it reaches a normal adult level at 14 or 15", because "children have less glycogen stored in their muscles, and anaerobic glycolysis enzymes are less active" (Maglischo, 1993, p. 253).

Marinho, Amorim, Costa and Neiva (2011) studied the relationship between anaerobic critical velocity and short-distance performances in the four swimming strokes in young swimmers and concluded that linear relationships between anaerobic critical velocity and performance in the 100-meter event have been found, which indicates that anaerobic performance components are important in young swimmers.

Olbrecht (2013) notices that "lactate tests can be carried out regardless of the age of the swimmer and it would be wrong to determine for whom lactate tests might be meaningful on the basis of age alone" (p. 2028). Most young swimmers are unable to produce large amounts of lactate, but those individuals who are able to do so should use this skill to improve their training programme.

\section{Research questions}

We formulated the following hypothesis for this study: Biological parameters are similar in prepubertal and pubertal swimmers when performing an anaerobic effort.

\section{Purpose of the study}

This research paper is conducted to compare physiological variables of prepubertal and pubertal swimmers after an anaerobic capacity test with the aim to determine if training-based adaptations are similar.

\section{Material and Method}

In this study, we used the ascertaining pedagogical experiment with two variables (the prepubertal group and the pubertal group), controlled observation, the Borg Scale, graphical method, mathematical and statistical method.

\section{Subjects}

We conducted this research at the Aqua Team Sports Club. Two groups of young swimmers (boys and girls) with ages between 10 and 14 were selected. Swimmers had different levels of performance (including national champions and national record holders), but all of them were involved in a sustained training programme for at least two or three years. The training programme has similar periodisation and specific training stimuli for the subjects in both groups, with the adjustments demanded by their physical capabilities.

The first group had 10 prepubertal swimmers, and the second group had 10 pubertal swimmers, with each group equally divided in 5 boys and 5 girls.

\section{The 100-meter time trial}

Stager, Stickford and Grand (2015) highlight that "no definitive markers or tests can be employed to quantify the capacity of nonaerobic pathways. [...] Thus, most tests used to assess anaerobic capacity are performance tests during which athletes are asked to perform a task. Results are then compared with those of other athletes, as well as past performances" (p. 163). 
With these arguments, we selected a 100-meter time trial in order to assess the anaerobic effort capacity. The subjects can swim the distance in a time that fits the profile of the anaerobic energy pathway.

The swimmers were instructed to swim 100-meter freestyle with maximum effort. After having finished the swim, they were asked to come out of the water for their blood samples to be collected.

After the first blood sample was collected, the swimmer remained sited for fifteen minutes while two more blood samples were taken in the fifth and twelfth minute after abruptly ending the swim.

The subjects were asked to rate their effort using the Borg Scale for perceived exertion.

\section{Equipment used}

We used the Lactate Meter Plus from Nova Biomedical to measure the concentration of blood lactate in the body. The device is characterised by a high-speed of analysis and a maximum deviation of $0.2 \mathrm{mmol}$.

The subject must clean and dry his or her fingertip before procedure. After making the puncture in their finger skin with a very thin, least painful lance, the first drop of blood that comes out is wiped off and the second drop is poured on the lactate strip. The unit automatically points the blood lactate concentration within the next 13 seconds. Measurements were made in collaboration with the National Institute for Sport Research.

\section{Mathematical and statistical methods}

For this research, we chose to use the following mathematical and statistical indices:

- the mean - parameter of central tendency;

- the standard deviation - statistical parameter of dispersion.

\section{Results}

Each swimmer was tested separately on an empty pool lane after receiving detailed instructions about the testing protocol.

The basal value of blood lactate was measured before the time trial, and the mean values for both groups were calculated. We observed that the prepubertal group had a mean of $1.7 \mathrm{mmol}$, and the pubertal group had 1.56 mmol. The $0.14 \mathrm{mmol}$ difference between the means is of small significance.

We synthesised the results in the following tables. The prepubertal group is shown in Table 1, and the pubertal group is shown in Table 2.

Table 1. Summary table with the evolution of the measured physiological variables for prepubertal swimmers

\begin{tabular}{lllllcccc}
\hline & \multirow{2}{*}{$\begin{array}{c}\text { Name } \\
\text { initials }\end{array}$} & Age & Gender & & \multicolumn{6}{c}{ Results } \\
\cline { 7 - 9 } & & & Basal & \multirow{2}{*}{ Borg } & \multicolumn{3}{c}{ Measured values of lactate (mmol) } \\
\cline { 7 - 9 } & B. R. & 10 & M & 1.6 & 20 & 5.3 & 3.8 & 3 \\
2 & S.D. & 10 & M & 1.8 & 18 & 6.3 & 9.4 & 4.7 \\
3 & S. T. & 10 & M & 1.6 & 19 & 7.7 & 5.2 & 4.1 \\
4 & I. A. & 11 & M & 1.4 & 19 & 8 & 6.1 & 3.9 \\
5 & B. A. & 10 & F & 1.9 & 12 & 6.3 & 5.9 & 3.3 \\
6 & C.C. & 11 & F & 1.1 & 15 & 8.5 & 6 & 4.5 \\
7 & R.C. & 11 & F & 2.1 & 18 & 7.9 & 5.6 & 4.2 \\
8 & C.A. & 12 & F & 2.2 & 18 & 6 & 4.9 & 4.3 \\
9 & M. I. & 11 & F & 1.3 & 17 & 5 & 2.9 & 1.8 \\
10 & P.A. & 12 & M & 2 & 19 & 3.3 & 2.4 & 2 \\
& Arithmetic mean & & $\mathbf{1 . 7 0}$ & $\mathbf{1 7 . 5 0}$ & $\mathbf{6 . 4 3}$ & $\mathbf{5 . 2 2}$ & $\mathbf{3 . 5 8}$ \\
\multicolumn{3}{l}{ Standard deviation } & & $\mathbf{0 . 3 4}$ & $\mathbf{2 . 2 5}$ & $\mathbf{1 . 5 4}$ & $\mathbf{1 . 8 7}$ & $\mathbf{0 . 9 7}$ \\
\hline
\end{tabular}

In Table 1, we can see that the values recorded in the first minute range from $3.3 \mathrm{mmol}$ to $8.5 \mathrm{mmol}$. In the fifth minute, the values range from $2.4 \mathrm{mmol}$ to $9.4 \mathrm{mmol}$. The third measurement reveals a restraining range of values from $1.8 \mathrm{mmol}$ to $4.7 \mathrm{mmol}$.

The general evolution of lactate measurements can be observed in Figure 1. 


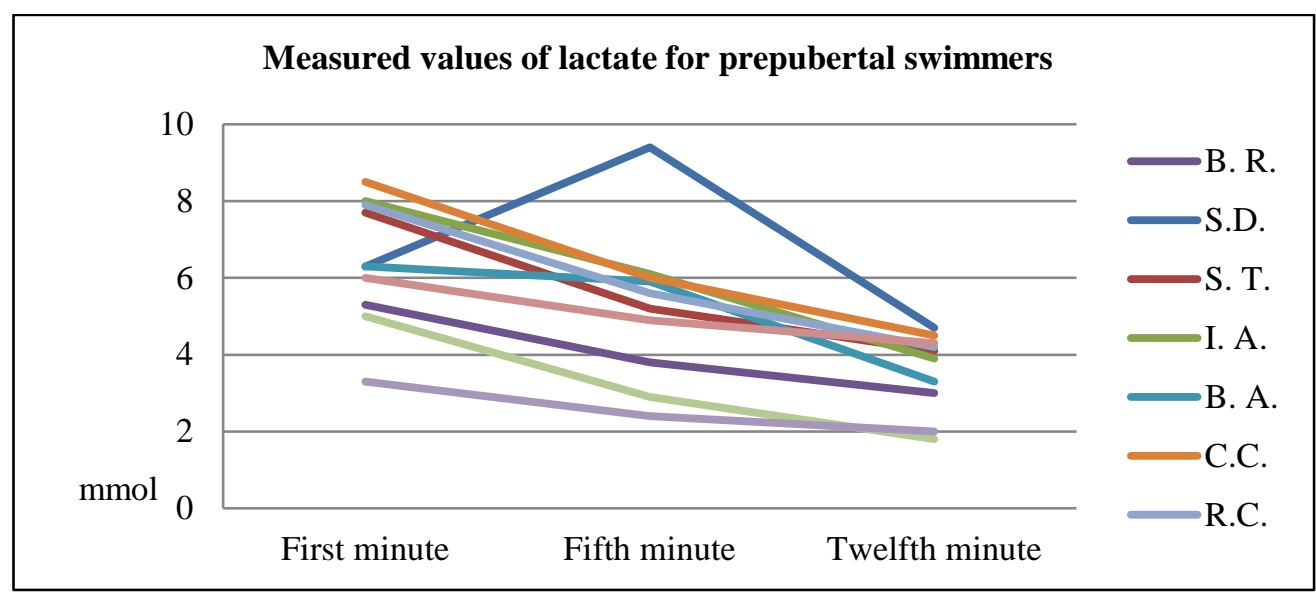

Figure 1. Graphical representation of the measured values of lactate for prepubertal group (mmol)

We summarised the values of blood lactate for the pubertal group in Table 2.

The values in the first minute are scaled from $3.1 \mathrm{mmol}$ to $8.7 \mathrm{mmol}$, while the fifth-minute measurements are scaled 2.4 to $6.7 \mathrm{mmol}$.

The final sampling revealed a minimum value of $2.1 \mathrm{mmol}$ and a maximum value of $5.7 \mathrm{mmol}$.

The general evolution of lactate measurements can be observed in Figure 2.

Table 2. Summary table with the evolution of the measured physiological variables for pubertal swimmers

\begin{tabular}{|c|c|c|c|c|c|c|c|c|}
\hline \multirow{3}{*}{ No. } & \multirow{3}{*}{$\begin{array}{l}\text { Name } \\
\text { initials }\end{array}$} & \multirow{3}{*}{ Age } & \multirow{3}{*}{ Gender } & \multicolumn{5}{|c|}{ Results } \\
\hline & & & & \multirow{2}{*}{ Basal } & \multirow{2}{*}{ Borg } & \multicolumn{3}{|c|}{ Measured values of lactate (mmol) } \\
\hline & & & & & & First minute & Fifth minute & Twelfth minute \\
\hline 1 & I.D. & 13 & $\mathrm{~F}$ & 2 & 17 & 8 & 6.5 & 4.2 \\
\hline 2 & P.D. & 13 & M & 1 & 18 & 4.6 & 3.6 & 3.1 \\
\hline 3 & P.D. & 13 & M & 2 & 16 & 7.7 & 3.6 & 3.6 \\
\hline 4 & C.L. & 14 & M & 1.5 & 16 & 8.7 & 6.7 & 5 \\
\hline 5 & N.A & 13 & $\mathrm{~F}$ & 1.6 & 20 & 7.9 & 6.1 & 5.7 \\
\hline 6 & I.B. & 13 & $\mathrm{~F}$ & 1.5 & 19 & 3.1 & 2.4 & 2.1 \\
\hline 7 & N.I. & 13 & M & 2.2 & 17 & 5.9 & 3.6 & 2.7 \\
\hline 8 & S.E. & 14 & $\mathrm{~F}$ & 1 & 17 & 4.2 & 3.2 & 2.6 \\
\hline 9 & G.D. & 13 & M & 1.5 & 18 & 6.1 & 5.5 & 4 \\
\hline 10 & L.C. & 13 & M & 1.3 & 19 & 7.9 & 5.6 & 4.2 \\
\hline \multicolumn{4}{|c|}{ Arithmetic mean } & 1.56 & 17.7 & 6.41 & 4.68 & 3.72 \\
\hline \multicolumn{4}{|c|}{ Standard deviation } & 0.39 & 1.27 & 1.83 & 1.48 & 1.07 \\
\hline
\end{tabular}

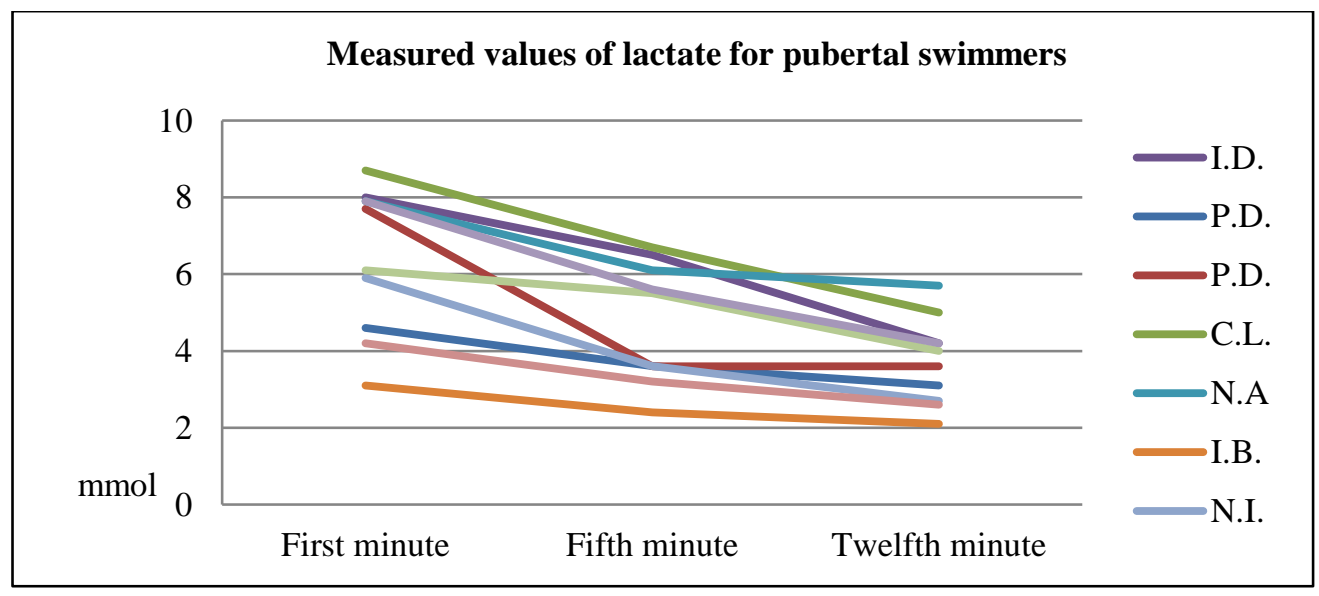


Figure 2. Graphical representation of the measured values of lactate for pubertal group (mmol) We graphically represented the results of both groups in Figure 3.

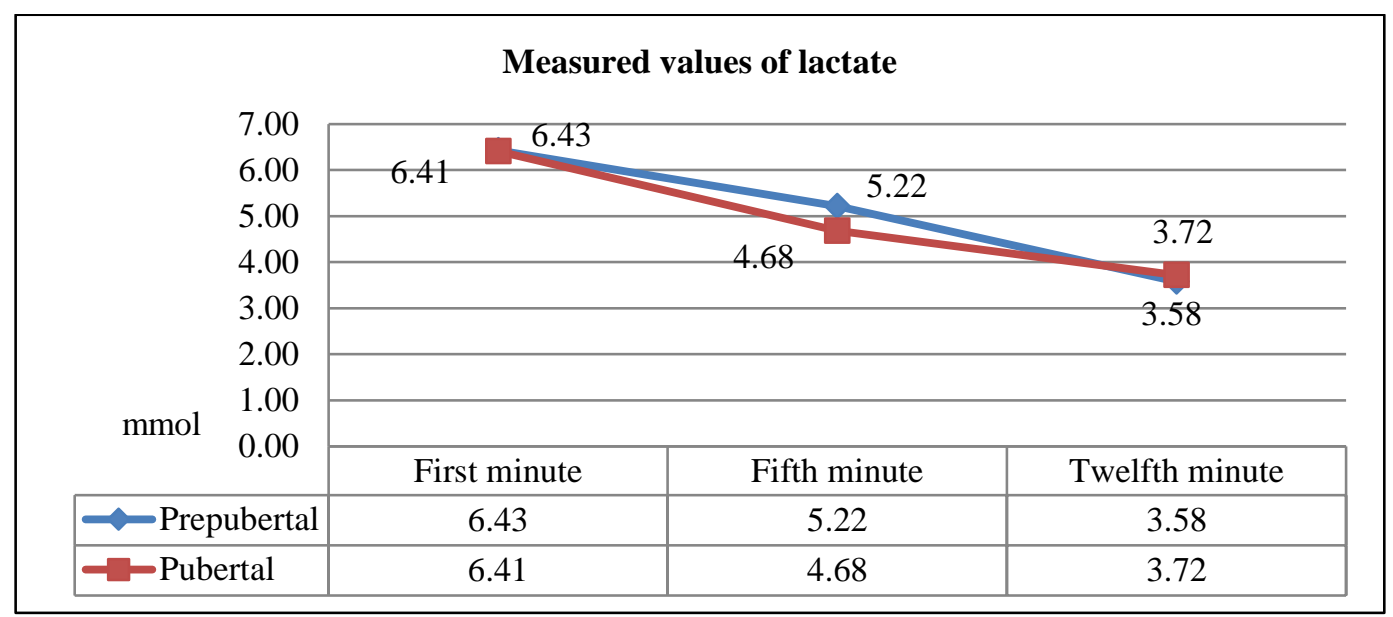

Figure 3. Graphical representation of the measured values of lactate (mmol)

The blood lactate values were recorded with the Lactate Meter Plus before the test and after it, namely in the first, fifth and twelfth minute.

In the first minute, the mean value of blood lactate for the prepubertal group was $6.43 \mathrm{mmol}$ and $6.41 \mathrm{mmol}$ for the pubertal group. We determined through calculation that, in the first minute, the difference was $0.3 \%$ between the two groups, indicating a nearly identical reaction of the physiological value.

There was a difference of $0.54 \mathrm{mmol}$ in the fifth minute between the two groups. Prepubertal swimmers had a higher value by $11.53 \%$. This could be possible due to a larger amount of enzymes that cleaned blood lactate.

The third measurement showed that the prepubertal group had a smaller value with $3.91 \%$, as we measured $3.58 \mathrm{mmol}$ for the prepubertal group and $3.72 \mathrm{mmol}$ for the pubertal group. The difference between the groups is insignificant, yet the prepubertal group had recovered faster from the fifth minute to the twelfth minute.

According to the calculated standard deviations, all the means for the measured physiological variables of both groups show a normal distribution of data.

After analysing the values with which the swimmers rated the level of perceived exertion, we observed that the means were similar to some extent, with 17.70 for the prepubertal group and 17.50 for the pubertal group.

\section{Conclusion}

The following conclusions were formulated after interpreting the results:

- Prepubertal and pubertal swimmers have the same value of blood lactate immediately after the test.

- Pubertal swimmers better diminish the amount of blood lactate after five minutes, and prepubertal swimmers have better recovery from the fifth to the twelfth minute.

- Anaerobic capacity is a key element in achieving competition success.

- Although aerobic capacity training should be the focus for age-group swimmers, coaches should not avoid exposing them to reasonable amounts of anaerobic training.

- The aim of anaerobic sets should be to familiarise the swimmers with high-intensity efforts and to gain information that can help guide the development plan.

- Prepubertal and pubertal swimmers have a similar reaction to anaerobic effort testing - the research hypothesis is confirmed.

\section{Authors' Contributions}

All authors contributed equally to this study and should be considered as main authors. 


\section{References}

Brooks, M. (2011). Developing swimmers. Champaign, IL: Human Kinetics.

Maglischo, E. (1993). Swimming even faster. Mountain View, California: Mayfield.

Marinho, D. A., Amorim, R. A., Costa, A. M., \& Neiva, H. P. (2011). The relationship between "anaerobic" critical velocity and swimming performance in young swimmers. Medicine \& Science in Sports \& Exercise, 43(5), 665-666. doi:10.4100/jhse.2011.61.09

Olbrecht, J. (2013). The science of winning: Planning, periodizing and optimizing swim training. Tienen: F\&G Partners.

Stager, Stickford, J., \& Grand, K. (2015). Energy systems and physiology. In S. Riewald \& S. Rodeo (Eds.), Science of swimming faster: The training, technology and evolution of extraordinary performance (Chapter 8, pp. 147-172). Champaign, IL: Human Kinetics.

Sweetenham, B., \& Atkinson, J. (2003). Championship swim training. Champaign, IL: Human Kinetics. 